\title{
16. OXYGEN AND CARBON ISOTOPE STRATIGRAPHY OF DEEP SEA DRILLING PROJECT HOLE 552A: PLIO-PLEISTOCENE GLACIAL HISTORY ${ }^{1}$
}

\author{
N. J. Shackleton and M. A. Hall, University of Cambridge ${ }^{2}$
}

\begin{abstract}
Oxygen and carbon isotope ratios in benthic foraminifers have been determined at $10 \mathrm{~cm}$ intervals through the top $59 \mathrm{~m}$ of DSDP Hole 552A. This provides a glacial record of remarkable resolution for the late Pliocene and Pleistocene. The major glacial event which marked the onset of Pleistocene-like glacial-interglacial alternations was at about $2.4 \mathrm{~m} . \mathrm{y}$. ago. These very high-resolution data do not support the notion of significant Northern Hemisphere glaciation between 3.2 and 2.4 m.y. ago.
\end{abstract}

\section{INTRODUCTION}

Until recently it has been impossible to study the deepsea sediment record of the onset of glaciations with the same stratigraphic resolution as is available for the middle and late Pleistocene glacial sequence. The best oxygen isotope records of the past 150 thousand years (k.y.) are from piston cores in areas where accumulation rates are typically 2 to $4 \mathrm{~cm} / \mathrm{k}$.y.; the limitations of piston coring techniques preclude longer records being obtained. Shackleton and Hall (1983) have obtained a detailed oxygen isotope record from Deep Sea Drilling Project (DSDP) Site 504, one of the first sites to be cored with the HPC, but although this provided a very good record, its usefulness was limited by the significant between-core gaps.

DSDP Hole 552A was cored on the west flank of Rockall Plateau (Fig. 1) at a water depth of $2311 \mathrm{~m}$. This puts the site in a good position for monitoring the dramatic glacial-interglacial changes that have characterized the Pleistocene (Ruddiman and McIntyre, 1976). Moreover its location is particularly interesting from the standpoint of a European Quaternary geologist; our notion of the nature and timing of early glacial events is extremely vague: thus, a good record so close to our coasts is particularly valuable. Hole $552 \mathrm{~A}$ was cored with exceptionally high recovery and minimal core disturbance; the varied nature of the sediment revealed any coring disturbance very clearly (Core 6 was obviously disturbed and was not sampled for this study). Because of these advantages, we devoted considerable attention to the site despite the disadvantages of the region. The chief of these is that the nature of the sediment varies dramatically between ice-rafted debris and foraminiferal ooze; a uniform rate of sedimentation is unlikely. On the other hand, this feature does give the record a special interest; heavy glacial debris accumulation on Rockall has clear implications for climate on the nearby British Isles.

\footnotetext{
${ }^{1}$ Roberts, D. G., Schnitker, D., et al., Init. Repts. DSDP, 81: Washington (U.S. Govt. Printing Office)

2 Address: Godwin Laboratory for Quaternary Research, University of Cambridge, Free School Lane, Cambridge CB2 3RS, United Kingdom.
}

\section{METHODS}

The sediment was dispersed by shaking it for a few hours in distilled water, and sieved on a $150 \mu \mathrm{m}$ screen. The portion retained was dried at $60^{\circ} \mathrm{C}$ and weighed. The fine fraction was then settled for 24 hr., the water siphoned off to within about $1 \mathrm{~cm}$ of the sediment, and the fine residue dried and weighed. The fine fraction was retained for nannofossil studies.

Because we have in the past obtained apparently reliable isotopic data from Uvigerina spp., from Cibicidoides wuellerstorfi, and from Globocassidulina subglobosa, we planned to select for analysis whichever of these species was most abundant in each sample. In addition, a number of duplicates were analyzed in order to build up our knowledge of the isotopic relationships between these species. In a small number of samples containing insufficient of these three, other species, or mixtures, were analyzed; a few other species were analyzed solely in order to gain information on interspecific isotopic differences. Standard techniques for analysis were used: reaction with $100 \%$ orthophosphoric acid at $50^{\circ} \mathrm{C}$, removal of water, and isotopic analysis in a VG Isogas 903 triple collector mass spectrometer. A minor but significant modification to the procedure described by Shackleton, Imbrie, and Hall (1983) is that an electric cooler running at between $-90^{\circ}$ and $-94^{\circ} \mathrm{C}$ was used to remove water vapor instead of dry ice $\left(-78^{\circ} \mathrm{C}\right)$. This eliminates problems with occasional $\mathrm{CO}_{2}$ samples contaminated by water. Overall analytical uncertainty during the time these measurements were made was $\pm 0.07 \%\left({ }^{18} \mathrm{O}\right)$ and $\pm 0.05 \%\left({ }^{13} \mathrm{C}\right)$ (both 1-sigma values). All analytical values are given in Table 1. The calibrations in Table 2 were used to adjust values for the various species analyzed in Table 1 towards isotopic equilibrium values (for ${ }^{18} \mathrm{O}$ ) and towards an estimated ${ }^{13} \mathrm{C}$ content of dissolved $\mathrm{CO}_{2}$ (for ${ }^{13} \mathrm{C}$ ). Where more than one analysis was made in a sample, a mean was taken after species adjustment.

\section{OXYGEN ISOTOPE RECORD}

In Figures 2 to 4 , the oxygen and carbon isotope records are shown as a function of approximate estimated age. A simple time scale is used, based on the paleomagnetic data of Kent (see Zimmerman et al., this volume); the control points used are given in Table 3, and age was estimated by linear interpolation between these points. In the upper part of the record (Fig. 2), the well-known features of the oxygen isotope record of the past million years (Stages 1 to 23 ) may be recognized with relative ease. For this part of the section, the global oxygen isotope record is relatively well known, and the chief value of such a record for Hole 552A lies in the possibility of accurate correlation to other regions. However, it should be noted that this is the first published ${ }^{18} \mathrm{O}$ record of the whole middle Pleistocene glacial record based on the 


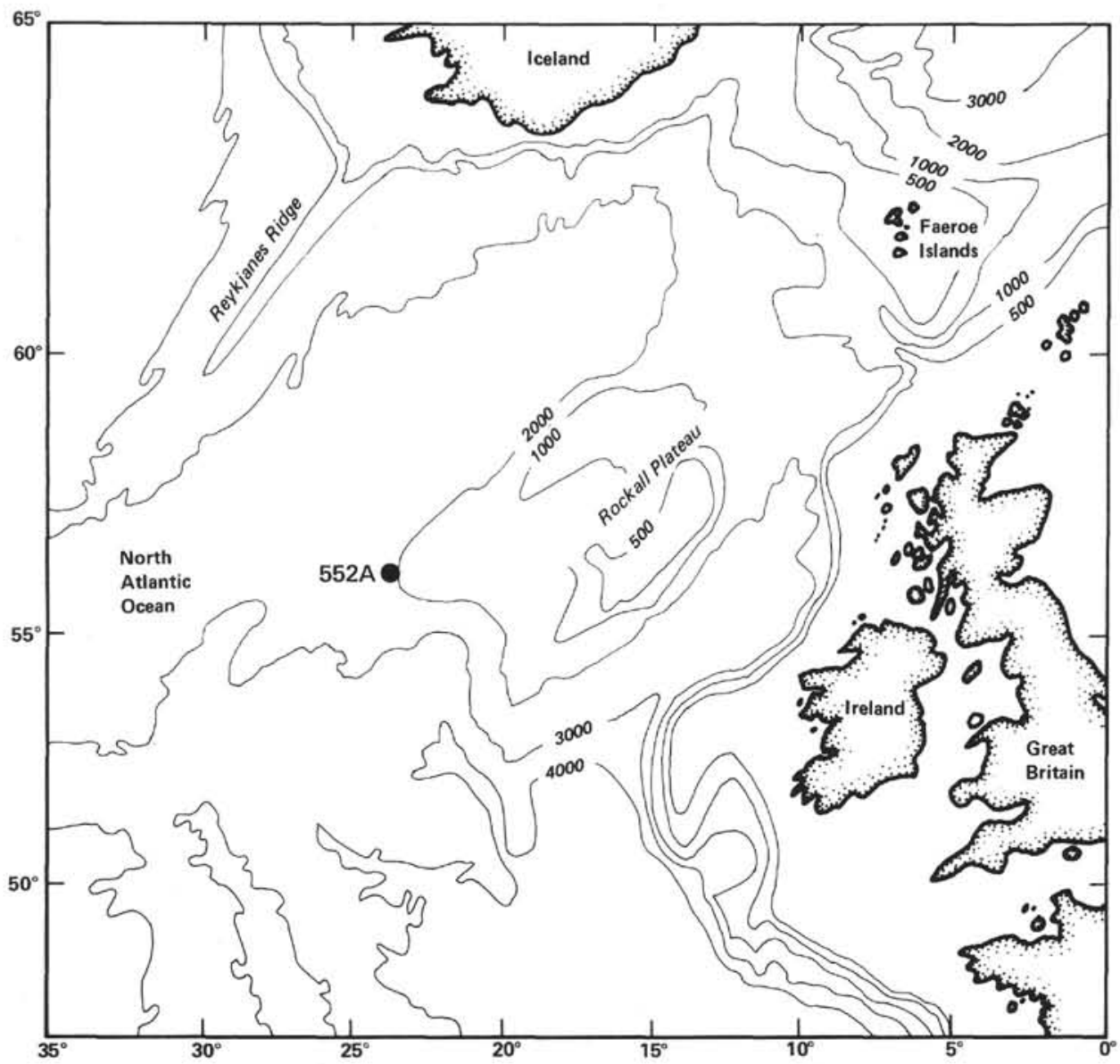

Figure 1. Location map for Hole $552 \mathrm{~A}\left(56^{\circ} 02.56^{\prime} \mathrm{N}, 23^{\circ} 13.39^{\prime} \mathrm{W}\right.$, water depth $\left.2311 \mathrm{~m}\right)$.

Table 1. Oxygen and carbon isotope data for Hole 552A. (Depths are consistent with the site chapter, this volume.)

\begin{tabular}{|c|c|c|c|}
\hline $\begin{array}{l}\text { Depth } \\
\text { (m) }\end{array}$ & Species & $\delta^{18} \mathrm{O}$ & $\delta^{13} \mathrm{C}$ \\
\hline 0.10 & Cibicidoides wuellerstorfi & 3.08 & 1.26 \\
\hline 0.20 & Cibicidoides wuellerstorfi & 2.71 & 1.29 \\
\hline 0.30 & Cibicidoides wuellerstorfi & 3.64 & 1.09 \\
\hline 0.30 & Hoeglundina elegans & 4.53 & 2.34 \\
\hline 0.39 & Cibicidoides wuellerstorfi & 4.09 & 0.94 \\
\hline 0.39 & Hoeglundina elegans & 4.59 & 2.19 \\
\hline 0.51 & Cibicidoides wuellerstorfi & 3.99 & 1.05 \\
\hline 0.62 & Cibicidoides wuellerstorfi & 4.08 & 1.11 \\
\hline 0.71 & Cibicidoides wuellerstorfi & 3.77 & 1.24 \\
\hline 0.78 & Cibicidoides wuellerstorfi & 3.91 & 1.16 \\
\hline 0.89 & Cibicidoides wuellerstorfi & 3.97 & 1.21 \\
\hline 1.00 & Cibicidoides wuellerstorfi & 4.00 & 1.10 \\
\hline 1.10 & Cibicidoides wuellerstorf $i$ & 3.91 & 0.91 \\
\hline 1.20 & Cibicidoides wuellerstorfi & 3.93 & 1.13 \\
\hline 1.32 & Cibicidoides wuellerstorfi & 3.59 & 1.14 \\
\hline 1.37 & Cibicidoides wuellerstorfi & 3.69 & 1.25 \\
\hline 1.37 & Hoeglundina elegans & 5.26 & 2.59 \\
\hline 1.37 & Cibicidoides wuellerstorfi & 3.67 & 1.27 \\
\hline 1.40 & Cibicidoides wuellerstorfi & 3.80 & 0.99 \\
\hline 1.42 & Cibicidoides wuellerstorfi & 3.72 & 0.93 \\
\hline 1.51 & Cib. and Melonis spp. & 3.97 & 0.55 \\
\hline 1.62 & Cibicidoides wuellerstorfi & 3.92 & 1.11 \\
\hline 1.63 & Cibicidoides wuellerstorfi & 3.50 & 1.38 \\
\hline 1.70 & Cibicidoides wuellerstorfi & 3.49 & 1.30 \\
\hline 1.73 & Cibicidoides wuellerstorf $i$ & 3.48 & 1.37 \\
\hline 1.79 & Cibicidoides wuellerstorf $i$ & 3.39 & 1.28 \\
\hline
\end{tabular}

Table 1. (Continued).

\begin{tabular}{|c|c|c|c|}
\hline $\begin{array}{c}\text { Depth } \\
\text { (m) }\end{array}$ & Species & $\delta^{18} \mathrm{O}$ & $\delta^{13} \mathrm{C}$ \\
\hline 1.83 & Cibicidoides wuellerstorfi & 3.34 & 1.25 \\
\hline 1.90 & Cibicidoides wuellerstorfi & 3.39 & 1.31 \\
\hline 2.00 & Cibicidoides wuellerstorfi & 3.58 & 1.36 \\
\hline 2.11 & Cibicidoides wuellerstorfi & 2.84 & 1.23 \\
\hline 2.19 & Cibicidoides wuellerstor $f i$ & 2.55 & 1.11 \\
\hline 2.28 & Cibicidoides wuellerstorfi & 2.63 & 0.89 \\
\hline 2.40 & Cibicidoides wuellerstorfi & 2.98 & 0.61 \\
\hline 2.40 & Hoeglundina elegans & 4.51 & 2.23 \\
\hline 2.50 & Cibicidoides wuellerstor $f i$ & 4.05 & 0.78 \\
\hline 2.50 & Uvigerina sp. & 5.18 & -0.35 \\
\hline 2.50 & Hoeglundina elegans & 4.70 & 2.22 \\
\hline 2.60 & Cibicidoides wuellerstorfi & 4.36 & 0.78 \\
\hline 2.60 & Uvigerina sp. & 5.24 & -0.23 \\
\hline 2.70 & Cibicidoides wuellerstorf $i$ & 4.13 & 0.81 \\
\hline 2.70 & Uvigerina sp. & 4.90 & -0.18 \\
\hline 2.80 & Cibicidoides wuellerstorfi & 3.84 & 0.30 \\
\hline 2.90 & Cibicidoides wuellerstorfi & 4.29 & 0.75 \\
\hline 3.01 & Cibicidoides wuellerstorfi & 4.04 & 0.96 \\
\hline 3.10 & Melonis pompilliodes & 4.22 & 0.34 \\
\hline 3.10 & Uvigerina $\mathrm{sp}$. & 4.46 & -0.10 \\
\hline 3.20 & Cibicidoides wuellerstorfi & 3.88 & 0.82 \\
\hline 3.30 & Cibicidoides wuellerstorfi & 3.60 & 1.06 \\
\hline 3.38 & Cibicidoides wuellerstorfi & 3.34 & 1.23 \\
\hline 3.50 & Cibicidoides wuellerstorfi & 3.18 & 1.29 \\
\hline 3.60 & Cibicidoides wuellerstorfi & 3.29 & 1.19 \\
\hline 3.70 & Cibicidoides wuellerstorf $i$ & 3.30 & 1.10 \\
\hline
\end{tabular}


Table 1. (Continued).

\begin{tabular}{|c|c|c|c|}
\hline $\begin{array}{l}\text { Depth } \\
\text { (m) }\end{array}$ & Species & $\delta^{18} \mathrm{O}$ & $\delta^{13} \mathrm{C}$ \\
\hline 3.80 & Cibicidoides wuellerstorfi & 3.35 & 1.08 \\
\hline 3.90 & Cibicidoides wuellerstorfi & 3.32 & 0.99 \\
\hline 4.00 & Cibicidoides wuellerstorfi & 2.95 & 0.97 \\
\hline 4.40 & Cibicidoides wuellerstorfi & 2.97 & 1.01 \\
\hline 4.50 & Cibicidoides wuellerstorfi & 3.78 & 1.08 \\
\hline 4.60 & Cibicidoides wuellerstorf $i$ & 3.31 & 1.00 \\
\hline 4.70 & Cibicidoides wuellerstorf $i$ & 3.58 & 0.80 \\
\hline 4.80 & Cibicidoides wuellerstorfi & 3.62 & 1.29 \\
\hline 4.90 & Cibicidoides wuellerstorfi & 3.32 & 1.10 \\
\hline 4.98 & Cibicidoides wuellerstorfi & 3.91 & 1.13 \\
\hline 5.10 & Cibicidoides wuellerstorfi & 3.56 & 0.95 \\
\hline 5.20 & Cibicidoides wuellerstorfi & 3.47 & 1.22 \\
\hline 5.30 & Cibicidoides wuellerstorfi & 3.74 & 1.17 \\
\hline 5.39 & Cibicidoides wuellerstorfi & 2.98 & 0.98 \\
\hline 5.51 & Globocassidulina subglobosa & 3.09 & 0.32 \\
\hline 5.51 & Uvigerina sp. & 3.33 & -0.04 \\
\hline 5.51 & Cibicidoides wuellerstorfi & 2.57 & 1.14 \\
\hline 5.60 & Globocassidulina subglobasa & 3.23 & 0.49 \\
\hline 5.60 & Cibicidoides wuellerstorfi & 2.71 & 0.99 \\
\hline 5.72 & Cibicidoides wuellerstorf $i$ & 3.99 & 0.82 \\
\hline 5.80 & Cibicidoides wuellerstorfi & 3.86 & 1.18 \\
\hline 5.90 & Cibicidoides wuellerstorfi & 3.86 & 1.41 \\
\hline 6.00 & Cibicidoides wuellerstorfi & 3.74 & 1.30 \\
\hline 6.10 & Cibicidoides wuellerstorfi & 3.18 & 1.11 \\
\hline 6.20 & Cibicidoides wuellerstorfi & 2.86 & 1.39 \\
\hline 6.30 & Cibicidoides wuellerstorfi & 3.04 & 1.19 \\
\hline 6.40 & Cibicidoides wuellerstorfi & 2.82 & 1.37 \\
\hline 6.46 & Cibicidoides wuellerstorf $i$ & 2.63 & 1.28 \\
\hline 6.60 & Cibicidoides wuellerstorfi & 2.94 & 1.18 \\
\hline 6.70 & Cibicidoides wuellerstorfi & 3.00 & 0.99 \\
\hline 6.80 & Cibicidoides wuellerstorfi & 3.32 & 0.63 \\
\hline 6.90 & Cibicidoides wuellerstorfi & 3.39 & 0.88 \\
\hline 6.90 & Uvigerina sp. & 3.73 & -0.19 \\
\hline 7.01 & Cibicides bradyi & 3.52 & -0.29 \\
\hline 7.01 & Melonis spp. & 4.14 & -0.39 \\
\hline 7.10 & Melonis pompilliodes & 4.88 & 0.40 \\
\hline 7.30 & Hoeglundina elegans & 5.40 & 2.42 \\
\hline 7.30 & Cibicidoides wuellerstorfi & 4.28 & 0.81 \\
\hline 7.39 & Cibicidoides wuellerstorfi & 4.39 & 0.83 \\
\hline 7.39 & Uvigerina sp. & 4.62 & -0.13 \\
\hline 7.49 & Uvigerina sp. & 4.85 & -0.22 \\
\hline 7.60 & Uvigerina sp. & 4.72 & -0.14 \\
\hline 7.68 & Uvigerina sp. & 4.83 & -0.16 \\
\hline 7.80 & Uvigerina sp. & 4.67 & -0.30 \\
\hline 7.90 & Uvigerina sp. & 4.86 & 0.12 \\
\hline 8.00 & Uvigerina sp. & 4.75 & 0.10 \\
\hline 8.10 & Uvigerina $\mathrm{sp}$ : & 4.62 & 0.11 \\
\hline 8.19 & Uvigerina sp. & 4.81 & 0.02 \\
\hline 8.30 & Cibicidoides wuellerstorf $i$ & 3.92 & 0.97 \\
\hline 8.30 & Uvigerina $\mathrm{sp}$ & 4.91 & 0.11 \\
\hline 8.40 & Hoeglundina elegans & 5.22 & 2.39 \\
\hline 8.40 & Uvigerina sp. & 4.95 & 0.11 \\
\hline 8.53 & Cibicidoides wuellerstorf $i$ & 3.83 & 1.24 \\
\hline 8.60 & Cibicidoides wuellerstorf $i$ & 3.59 & 1.56 \\
\hline 8.70 & Cibicidoides spp. & 3.63 & 1.05 \\
\hline 8.80 & Cibicidoides wuellerstorfi & 3.36 & 1.64 \\
\hline 8.92 & Cibicidoides wuellerstorfi & 3.36 & 1.67 \\
\hline 9.00 & Cibicidoides wuellerstorfi & 3.26 & 1.74 \\
\hline 9.05 & Cibicidoides wuellerstorfi & 3.53 & 1.42 \\
\hline 9.15 & Cibicidoides wuellerstorfi & 3.88 & 1.12 \\
\hline 9.25 & Cibicidoides wuellerstorfi & 3.68 & 1.44 \\
\hline 9.35 & Cibicidoides wuellerstorfi & 3.72 & 0.96 \\
\hline 9.45 & Cibicidoides wuellerstorfi & 3.57 & 1.40 \\
\hline 9.50 & Cibicidoides wuellerstorfi & 3.58 & 1.49 \\
\hline 9.50 & Uvigerina sp. & 4.29 & 0.49 \\
\hline 9.60 & Cibicidoides wuellerstorfi & 4.13 & 1.19 \\
\hline 9.70 & Cibicidoides spp. & 3.81 & 1.19 \\
\hline 9.80 & Cibicidoides wuellerstorfi & 3.32 & 1.35 \\
\hline 9.90 & Cibicidoides wuellerstorf $i$ & 3.45 & 1.07 \\
\hline
\end{tabular}

Table 1. (Continued).

\begin{tabular}{|c|c|c|c|}
\hline $\begin{array}{l}\text { Depth } \\
\text { (m) }\end{array}$ & Species & $\delta^{18} \mathrm{O}$ & $\delta^{13} \mathrm{C}$ \\
\hline 10.00 & Cibicidoides wuellerstor $f i$ & 3.51 & 0.83 \\
\hline 10.10 & Cibicidoides wuellerstorfi & 3.34 & 0.95 \\
\hline 10.20 & Cibicidoides wuellerstorfi & 3.44 & 1.26 \\
\hline 10.30 & Cibicidoides wuellerstorfi & 3.30 & 1.12 \\
\hline 10.40 & Cibicidoides wuellerstorfi & 3.11 & 1.21 \\
\hline 10.50 & Cibicidoides wuellerstorfi & 3.39 & 1.01 \\
\hline 10.60 & Cibicidoides wuellerstorfi & 3.03 & 1.01 \\
\hline 10.60 & Melonis spp. & 3.27 & -0.76 \\
\hline 10.70 & Cibicidoides wuellerstorfi & 3.22 & 0.77 \\
\hline 10.80 & Cibicidoides spp. & 3.67 & 0.27 \\
\hline 10.90 & Cib. and Melonis spp. & 4.79 & 0.66 \\
\hline 11.00 & Cib. and Melonis spp. & 4.06 & 0.60 \\
\hline 11.10 & Cibicidoides spp. & 4.50 & 0.72 \\
\hline 11.20 & Cibicidoides wuellerstorfi & 4.41 & 0.77 \\
\hline 11.30 & Cibicidoides wuellerstorfi & 3.87 & 0.94 \\
\hline 11.40 & Cib. and Melonis spp. & 4.13 & 0.58 \\
\hline 11.50 & Cib. and Melonis spp. & 3.85 & 0.77 \\
\hline 11.60 & Cibicidoides wuellerstorfi & 4.07 & 1.02 \\
\hline 11.60 & Cassidulina carinata & 4.43 & -0.27 \\
\hline 11.60 & Melonis pompilloides & 4.23 & 0.31 \\
\hline 11.70 & Cibicidoides wuellerstorfi & 3.99 & 0.59 \\
\hline 11.70 & Melonis pompilliodes & 4.28 & -0.11 \\
\hline 11.80 & Cibicidoides spp. & 4.01 & 0.45 \\
\hline 11.80 & Melonis pompilliodes & 4.33 & 0.07 \\
\hline 11.90 & Cibicidoides wuellerstorfi & 4.01 & 0.76 \\
\hline 11.90 & Oridorsalis umbonifera & 4.50 & -1.01 \\
\hline 12.00 & Cibicidoides wuellerstorfi & 4.19 & 0.79 \\
\hline 12.10 & Cibicidoides wuellerstorf $i$ & 4.03 & 0.52 \\
\hline 12.20 & Cibicidoides wuellerstorf $i$ & 3.87 & 0.87 \\
\hline 12.30 & Cibicidoides wuellerstorfi & 3.52 & 0.85 \\
\hline 12.40 & Cibicidoides wuellerstorfi & 3.52 & 0.92 \\
\hline 12.50 & Cibicidoides wuellerstorfi & 3.37 & 1.38 \\
\hline 12.60 & Cibicidoides wuellerstorfi & 2.99 & 1.29 \\
\hline 12.70 & Cibicidoides wuellerstorfi & 3.35 & 1.22 \\
\hline 12.80 & Cibicidoides wuellerstorfi & 3.22 & 0.93 \\
\hline 12.90 & Cibicidoides wuellerstorfi & 3.58 & 0.69 \\
\hline 13.00 & Cibicidoides wuellerstorfi & 3.50 & 0.75 \\
\hline 13.10 & Cibicidoides wuellerstorfi & 3.52 & 1.14 \\
\hline 13.20 & Uvigerina sp. & 3.65 & 0.20 \\
\hline 13.30 & Cibicidoides wuellerstorfi & 4.07 & 1.20 \\
\hline 13.40 & Cibicidoides wuellerstorf $i$ & 3.84 & 1.08 \\
\hline 13.50 & Cibicidoides wuellerstorfi & 3.65 & 1.17 \\
\hline 13.60 & Cibicidoides wuellerstorfi & 3.43 & 1.09 \\
\hline 13.70 & Cibicidoides wuellerstorfi & 3.61 & 0.97 \\
\hline 13.80 & Mixed spp. & 3.97 & 0.42 \\
\hline 13.94 & Cassidulina carinata & 4.28 & -0.27 \\
\hline 14.06 & Cib. and Melonis spp. & 4.20 & 0.59 \\
\hline 14.20 & Cibicidoides wuellerstorfi & 3.07 & 1.11 \\
\hline 14.30 & Cibicidoides wuellerstorfi & 3.12 & 0.76 \\
\hline 14.40 & Cibicidoides wuellerstorfi & 3.09 & 1.10 \\
\hline 14.50 & Cibicidoides wuellerstorf $i$ & 3.54 & 0.14 \\
\hline 14.60 & Cibicidoides wuellerstorf $i$ & 4.05 & 0.27 \\
\hline 14.70 & Cibicidoides wuellerstorfi & 4.15 & 0.82 \\
\hline 14.78 & Cibicidoides wuellerstorfi & 3.80 & 0.69 \\
\hline 14.89 & Cibicidoides wuellerstorfi & 3.47 & 1.27 \\
\hline 15.00 & Cibicidoides spp. & 3.35 & 1.14 \\
\hline 15.00 & Melonis spp. & 3.39 & 0.40 \\
\hline 15.10 & Cibicidoides wuellerstorfi & 3.36 & 1.11 \\
\hline 15.20 & Cibicidoides wuellerstorfi & 3.21 & 1.01 \\
\hline 15.20 & Melonis spp. & 3.43 & 0.32 \\
\hline 15.30 & Cibicidoides wuellerstorfi & 3.28 & 0.90 \\
\hline 15.40 & Cibicidoides wuellerstorfi & 3.04 & 0.60 \\
\hline 15.50 & Cibicidoides wuellerstorfi & 3.20 & 0.81 \\
\hline 15.60 & Cibicidoides wuellerstorfi & 3.13 & 0.80 \\
\hline 15.70 & Cibicidoides wuellerstorfi & 3.21 & 0.12 \\
\hline 15.80 & Cibicidoides wuellerstorfi & 3.21 & 0.23 \\
\hline 15.89 & Cassidulina carinata & 4.83 & -0.55 \\
\hline 15.89 & Melonis barleanum & 4.47 & -0.98 \\
\hline 16.00 & Cassidulina carinata & 4.78 & -0.57 \\
\hline
\end{tabular}


Table 1. (Continued).

\begin{tabular}{|c|c|c|c|}
\hline $\begin{array}{l}\text { Depth } \\
\text { (m) }\end{array}$ & Species & $\delta^{18} \mathrm{O}$ & $\delta^{13} \mathrm{C}$ \\
\hline 16.00 & Cibicidoides wuellerstorfi & 3.88 & 0.45 \\
\hline 16.11 & Cibicidoides wuellerstorfi & 3.87 & -0.10 \\
\hline 16.20 & Cibicidoides wuellerstorfi & 3.36 & 0.19 \\
\hline 16.20 & Hoeglundina elegans & 4.98 & 1.83 \\
\hline 16.40 & Cibicidoides wuellerstorfi & 2.87 & -0.22 \\
\hline 16.40 & Stilostomella spp. & 4.42 & -1.25 \\
\hline 16.48 & Cib. and Melonis spp. & 4.13 & 0.40 \\
\hline 16.60 & Cibicidoides wuellerstorfi & 3.60 & 0.89 \\
\hline 16.70 & Cibicidoides wuellerstorfi & 3.51 & 0.73 \\
\hline 16.79 & Cibicidoides wuellerstorfi & 3.54 & 0.67 \\
\hline 16.88 & Cibicidoides wuellerstorfi & 3.32 & 0.09 \\
\hline 16.99 & Hoeglundina elegans & 4.67 & 1.66 \\
\hline 17.20 & Cibicidoides wuellerstorfi & 3.82 & 0.66 \\
\hline 17.20 & Cassidulina carinata & 4.42 & -0.16 \\
\hline 17.30 & Cibicidoides wuellerstorfi & 3.30 & 0.88 \\
\hline 17.40 & Cibicidoides wuellerstorfi & 2.83 & 1.02 \\
\hline 17.49 & Cibicidoides wuellerstorf $i$ & 2.66 & 0.99 \\
\hline 17.60 & Cibicidoides wuellerstorfi & 2.86 & 0.83 \\
\hline 17.60 & Uvigerina sp. & 2.97 & 0.07 \\
\hline 17.69 & Cibicidoides wuellerstorfi & 3.60 & 0.20 \\
\hline 17.80 & Cibicidoides wuellerstorfi & 3.60 & 0.88 \\
\hline 17.90 & Cibicidoides wuellerstorfi & 3.53 & 1.03 \\
\hline 17.90 & Oridorsalis umbonifera & 4.18 & -0.41 \\
\hline 17.90 & Uvigerina sp. & 3.69 & 0.19 \\
\hline 18.00 & Cibicidoides wuellerstorfi & 3.18 & 0.84 \\
\hline 18.00 & Oridorsalis umbonifera & 3.76 & -0.41 \\
\hline 18.00 & Uvigerina sp. & 3.75 & 0.11 \\
\hline 18.10 & Cibicidoides wuellerstorfi & 3.19 & 1.11 \\
\hline 18.20 & Hoeglundina elegans & 3.97 & 2.14 \\
\hline 18.20 & Cib. and Melonis spp. & 3.74 & 0.37 \\
\hline 18.30 & Cibicidoides wuellerstorfi & 3.60 & 1.22 \\
\hline 18.42 & Cibicidoides wuellerstorfi & 3.39 & 1.26 \\
\hline 18.55 & Cib. and Melonis spp. & 3.43 & 0.77 \\
\hline 18.75 & Cibicidoides wuellerstorfi & 3.77 & 1.11 \\
\hline 18.85 & Uvigerina sp. & 3.74 & 0.27 \\
\hline 18.85 & Cibicidoides wuellerstorfi & 3.45 & 1.10 \\
\hline 18.95 & Cibicidoides wuellerstorfi & 3.27 & 1.08 \\
\hline 18.95 & Uvigerina sp. & 3.96 & 0.23 \\
\hline 19.03 & Cibicidoides wuellerstorf $i$ & 3.48 & 1.10 \\
\hline 19.03 & Uvigerina sp. & 3.71 & 0.06 \\
\hline 19.10 & Cibicidoides wuellerstorfi & 3.41 & 1.08 \\
\hline 19.20 & Cibicidoides wuellerstorfi & 3.68 & 1.05 \\
\hline 19.29 & Cibicidoides wuellerstorfi & 3.21 & 1.21 \\
\hline 19.29 & Uvigerina sp. & 3.90 & 0.43 \\
\hline 19.40 & Cibicidoides wuellerstorfi & 3.65 & 1.05 \\
\hline 19.48 & Cibicidoides wuellerstorfi & 3.79 & 0.98 \\
\hline 19.59 & Cibicidoides wuellerstorfi & 3.75 & 0.84 \\
\hline 19.70 & Cibicidoides wuellerstorfi & 3.67 & 0.73 \\
\hline 19.80 & Cibicidoides wuellerstorf $i$ & 2.94 & 1.16 \\
\hline 19.80 & Uvigerina sp. & 3.83 & 0.29 \\
\hline 19.90 & Uvigerina sp. & 3.25 & 0.41 \\
\hline 20.00 & Cibicidoides wuellerstorfi & 2.82 & 1.17 \\
\hline 20.00 & Uvigerina sp. & 3.54 & 0.14 \\
\hline 20.08 & Cibicidoides wuellerstorfi & 3.09 & 1.20 \\
\hline 20.08 & Uvigerina sp. & 3.39 & 0.12 \\
\hline 20.19 & Cibicidoides wuellerstorfi & 3.12 & 1.13 \\
\hline 20.19 & Uvigerina sp. & 3.78 & -0.08 \\
\hline 20.30 & Cibicidoides wuellerstorfi & 3.43 & 1.19 \\
\hline 20.30 & Uvigerina sp. & 4.12 & -0.07 \\
\hline 20.38 & Uvigerina sp. & 4.14 & -0.21 \\
\hline 20.48 & Cibicidoides wuellerstorfi & 3.74 & 0.97 \\
\hline 20.48 & Uvigerina sp. & 4.09 & 0.02 \\
\hline 20.60 & Cibicidoides wuellerstorfi & 3.61 & 0.99 \\
\hline 20.70 & Cibicidoides wuellerstorfi & 3.35 & 1.06 \\
\hline 20.79 & Cibicidoides wuellerstorfi & 3.60 & 0.74 \\
\hline 20.90 & Cibicidoides spp. & 3.89 & -0.05 \\
\hline 21.00 & Cibicidoides wuellerstorfi & 3.96 & 0.81 \\
\hline 21.09 & Cibicidoides wuellerstorfi & 3.81 & 0.80 \\
\hline 21.19 & Cibicidoides wuellerstorfi & 3.60 & 0.86 \\
\hline
\end{tabular}

Table 1. (Continued).

\begin{tabular}{|c|c|c|c|}
\hline $\begin{array}{l}\text { Depth } \\
\text { (m) }\end{array}$ & Species & $\delta^{18} \mathrm{O}$ & $\delta^{13} \mathrm{C}$ \\
\hline 21.30 & Cibicidoides wuellerstorfi & 3.27 & 0.99 \\
\hline 21.38 & Uvigerina sp. & 3.68 & -0.14 \\
\hline 21.40 & Cibicidoides wuellerstorfi & 3.15 & 1.29 \\
\hline 21.48 & Uvigerina sp. & 3.49 & -0.17 \\
\hline 21.50 & Cibicidoides wuellerstorf $i$ & 2.91 & 1.03 \\
\hline 21.60 & Cibicidoides wuellerstorfi & 3.02 & 1.12 \\
\hline 21.69 & Cibicidoides wuellerstorfi & 2.88 & 1.09 \\
\hline 21.79 & Cibicidoides wuellerstorfi & 3.61 & 0.98 \\
\hline 21.88 & Cibicidoides wuellerstorfi & 4.06 & 1.12 \\
\hline 21.99 & Cibicidoides wuellerstorfi & 4.09 & 0.99 \\
\hline 22.10 & Cibicidoides wuellerstorfi & 3.32 & 0.87 \\
\hline 22.15 & Uvigerina sp. & 3.85 & 0.22 \\
\hline 22.20 & Cibicidoides wuellerstorfi & 2.76 & 0.93 \\
\hline 22.24 & Uvigerina sp. & 4.08 & -0.09 \\
\hline 22.26 & Cibicidoides wuellerstorfi & 3.37 & 1.14 \\
\hline 22.38 & Cibicidoides wuellerstorfi & 2.85 & 1.20 \\
\hline 22.48 & Cibicidoides wuellerstorfi & 2.70 & 0.89 \\
\hline 22.58 & Cibicidoides wuellerstorfi & 2.46 & 1.02 \\
\hline 22.69 & Cibicidoides wuellerstorfi & 3.89 & 1.05 \\
\hline 22.78 & Cibicidoides wuellerstorfi & 3.73 & 1.07 \\
\hline 22.85 & Cibicidoides wuellerstorfi & 3.70 & 0.86 \\
\hline 22.94 & Cibicidoides wuellerstorfi & 3.36 & 1.07 \\
\hline 23.05 & Cibicidoides wuellerstorfi & 3.31 & 1.22 \\
\hline 23.15 & Cibicidoides wuellerstorf $i$ & 3.04 & 1.23 \\
\hline 23.24 & Cibicidoides wuellerstorfi & 2.72 & 0.97 \\
\hline 23.31 & Uvigerina sp. & 4.19 & -0.20 \\
\hline 23.42 & Cibicidoides wuellerstorfi & 3.50 & 0.90 \\
\hline 23.49 & Cibicidoides wuellerstorfi & 3.48 & 0.84 \\
\hline 23.59 & Cibicidoides wuellerstorfi & 3.43 & 0.76 \\
\hline 23.69 & Cibicidoides wuellerstorfi & 3.33 & 0.90 \\
\hline 23.78 & Cibicidoides wuellerstorfi & 3.00 & 1.13 \\
\hline 23.88 & Cibicidoides wuellerstorfi & 3.28 & 1.01 \\
\hline 23.95 & Cibicidoides wuellerstorfi & 3.49 & 0.93 \\
\hline 29.10 & Cibicidoides wuellerstorfi & 2.82 & 1.12 \\
\hline 29.20 & Cibicidoides wuellerstorfi & 3.64 & 1.25 \\
\hline 29.33 & Cibicidoides wuellerstorfi & 3.05 & 1.19 \\
\hline 29.40 & Cibicidoides wuellerstorf $i$ & 2.92 & 1.31 \\
\hline 29.51 & Cibicidoides wuellerstorfi & 3.13 & 1.31 \\
\hline 29.57 & Cibicidoides wuellerstorfi & 3.00 & 1.33 \\
\hline 29.70 & Cibicidoides wuellerstorfi & 3.43 & 1.26 \\
\hline 29.80 & Cibiciodides wuellerstorfi & 3.53 & 1.22 \\
\hline 29.92 & Cibicidoides wuellerstorfi & 3.56 & 1.09 \\
\hline 29.92 & Uvigerina and Stilostomella & 3.99 & -0.19 \\
\hline 30.00 & Cibicidoides wuellerstorfi & 3.26 & 1.28 \\
\hline 30.10 & Cibicidoides wuellerstorfi & 3.18 & 1.26 \\
\hline 30.22 & Cibicidoides wuellerstorfi & 2.97 & 1.20 \\
\hline 30.30 & Cibicidoides wuellerstorfi & 3.07 & 1.00 \\
\hline 30.40 & Cibicidoides wuellerstorfi & 3.14 & 0.77 \\
\hline 30.51 & Stilostomella spp. & 3.89 & -0.03 \\
\hline 30.51 & Cibicidoides wuellerstorf $i$ & 3.06 & 0.08 \\
\hline 30.60 & Cibicidoides wuellerstorfi & 3.59 & 0.44 \\
\hline 30.70 & Cibicidoides wuellerstorf $i$ & 3.47 & 0.63 \\
\hline 30.79 & Cibicidoides wuellerstorfi & 3.23 & 0.54 \\
\hline 30.90 & Cibicidoides wuellerstorf $i$ & 3.12 & 0.94 \\
\hline 31.00 & Cibicidoides wuellerstorfi & 3.15 & 0.63 \\
\hline 31.10 & Cibicidoides wuellerstorfi & 3.16 & 0.64 \\
\hline 31.10 & Stilostomella spp. & 3.75 & -0.48 \\
\hline 31.20 & Cib. and Melonis spp. & 3.49 & -0.37 \\
\hline 31.27 & Cib. and Melonis spp. & 3.33 & -0.07 \\
\hline 31.40 & Cibicidoides wuellerstorfi & 3.36 & 0.74 \\
\hline 31.48 & Cib. and Melonis spp. & 3.82 & 0.18 \\
\hline 31.60 & Cibicidoides wuellerstorfi & 3.01 & 0.35 \\
\hline 31.69 & Cibicidoides wuellerstorfi & 2.70 & 1.08 \\
\hline 31.80 & Cibicidoides wuellerstorfi & 2.72 & 1.03 \\
\hline 31.80 & Melonis barleanum & 2.81 & -0.21 \\
\hline 31.90 & Cib. and Melonis spp. & 3.26 & -0.05 \\
\hline 31.99 & Cibicidoides wuellerstorfi & 2.87 & 0.87 \\
\hline 32.01 & Cibicidoides wuellerstorfi & 3.10 & 0.96 \\
\hline 32.10 & Cibicidoides wuellerstorfi & 3.22 & 0.95 \\
\hline
\end{tabular}


Table 1. (Continued).

\begin{tabular}{|c|c|c|c|}
\hline $\begin{array}{l}\text { Depth } \\
\text { (m) }\end{array}$ & Species & $\delta^{18} \mathrm{O}$ & $\delta^{13} \mathrm{C}$ \\
\hline 32.10 & Cibicidoides kullenbergi & 3.18 & 0.47 \\
\hline 32.20 & Cibicidoides wuellerstorfi & 3.07 & 0.70 \\
\hline 32.40 & Cibicidoides wuellerstorfi & 3.71 & 0.50 \\
\hline 32.40 & Melonis pompilliodes & 3.69 & 0.33 \\
\hline 32.40 & Melonis barleanum & 3.90 & -0.93 \\
\hline 32.50 & Cib. and Melonis spp. & 3.43 & -0.01 \\
\hline 32.50 & Stilostomella spp. & 4.00 & -0.20 \\
\hline 32.59 & Cibicidoides wuellerstorfi & 3.19 & 1.10 \\
\hline 32.70 & Cibicidoides wuellerstorfi & 1.54 & 1.08 \\
\hline 32.81 & Cibicidoides wuellerstorfi & 2.80 & 0.63 \\
\hline 32.91 & Cibicidoides wuellerstorfi & 2.93 & 0.26 \\
\hline 32.99 & Cibicidoides wuellerstorfi & 3.21 & 0.66 \\
\hline 33.10 & Cibicidoides wuellerstorfi & 3.47 & 0.88 \\
\hline 33.20 & Cibicidoides wuellerstorfi & 3.26 & 1.00 \\
\hline 33.30 & Cibicidoides wuellerstorfi & 3.20 & 1.18 \\
\hline 33.40 & Cibicidoides wuellerstorfi & 3.43 & 1.19 \\
\hline 33.51 & Cibicidoides wuellerstorfi & 3.12 & 1.12 \\
\hline 33.59 & Cibicidoides wuellerstorfi & 3.20 & 1.13 \\
\hline 33.70 & Cibicidoides wuellerstorfi & 2.99 & 1.21 \\
\hline 33.80 & Cibicidoides wuellerstorfi & 2.96 & 1.13 \\
\hline 34.00 & Cibicidoides wuellerstorfi & 3.01 & 0.79 \\
\hline 34.10 & Cibicidoides wuellerstorfi & 3.54 & 0.89 \\
\hline 34.20 & Cibicidoides wuellerstorfi & 3.11 & 1.09 \\
\hline 34.30 & Cibicidoides wuellerstorfi & 3.28 & 1.12 \\
\hline 34.40 & Cibicidoides wuellerstorfi & 3.02 & 0.85 \\
\hline 34.50 & Cibicidoides wuellerstorfi & 3.20 & 1.08 \\
\hline 34.60 & Cibicidoides wuellerstorfi & 2.94 & 1.04 \\
\hline 34.70 & Cibicidoides wuellerstorfi & 3.15 & 1.29 \\
\hline 34.80 & Cibicidoides wuellerstorfi & 2.96 & 1.24 \\
\hline 34.92 & Uvigerina sp. & 3.80 & 0.07 \\
\hline 35.00 & Cibicidoides wuellerstorfi & 3.32 & 0.88 \\
\hline 35.00 & Uvigerina sp. & 3.89 & -0.07 \\
\hline 35.10 & Uvigerina sp. & 3.93 & 0.16 \\
\hline 35.20 & Uvigerina sp. & 3.78 & -0.06 \\
\hline 35.30 & Uvigerina sp. & 3.99 & 0.30 \\
\hline 35.40 & Uvigerina sp. & 3.86 & 0.06 \\
\hline 35.50 & Uvigerina sp. & 3.59 & 0.37 \\
\hline 35.58 & Uvigerina sp. & 3.59 & 0.63 \\
\hline 35.58 & Cibicidoides wuellerstorfi & 2.93 & 1.49 \\
\hline 35.68 & Cibicidoides wuellerstorfi & 2.91 & 1.31 \\
\hline 35.78 & Cib. and Melonis spp. & 2.91 & 0.74 \\
\hline 35.89 & Cibicidoides wuellerstorfi & 2.82 & 0.78 \\
\hline 35.98 & Cibicidoides wuellerstorfi & 3.12 & 1.29 \\
\hline 35.98 & Cibicidoides wuellerstorfi & 3.04 & 1.36 \\
\hline 36.07 & Cibicidoides wuellerstorfi & 3.01 & 1.19 \\
\hline 36.29 & Cib. and Melonis spp. & 3.11 & 0.70 \\
\hline 36.38 & Cibicidoides wuellerstorfi & 3.21 & 1.32 \\
\hline 36.49 & Cibicidoides wuellerstorfi & 2.99 & 1.27 \\
\hline 36.58 & Cibicidoides wuellerstorfi & 3.04 & 1.34 \\
\hline 36.59 & Cibicidoides wuellerstorfi & 3.15 & 1.16 \\
\hline 36.78 & Cibicidoides wuellerstorfi & 3.34 & 1.10 \\
\hline 36.85 & Cibicidoides wuellerstorfi & 3.09 & 1.02 \\
\hline 36.98 & Cibicidoides wuellerstorfi & 3.87 & 0.96 \\
\hline 37.10 & Cibicidoides wuellerstorfi & 3.84 & 0.58 \\
\hline 37.20 & Cibicidoides spp. & 3.51 & 0.51 \\
\hline 37.30 & Cibicidoides wuellerstorfi & 3.66 & 0.60 \\
\hline 37.39 & Cibicidoides wuellerstorfi & 3.25 & 0.88 \\
\hline 37.39 & Uvigerina sp. & 3.92 & -0.82 \\
\hline 37.39 & Uvigerina sp. & 3.92 & 0.18 \\
\hline 37.50 & Cibicidoides wuellerstorfi & 3.13 & 0.44 \\
\hline 37.59 & Uvigerina sp. & 3.58 & 0.10 \\
\hline 37.59 & Cibicidoides wuellerstorfi & 2.92 & 0.82 \\
\hline 37.70 & Cibicidoides wuellerstorfi & 3.24 & 0.80 \\
\hline 37.79 & Cibicidoides spp. & 3.29 & 0.11 \\
\hline 37.90 & Cibicidoides wuellerstorfi & 3.00 & 0.98 \\
\hline 37.99 & Cibicidoides wuellerstorfi & 2.81 & 0.78 \\
\hline 38.10 & Cibicidoides wuellerstorfi & 3.91 & 0.67 \\
\hline 38.30 & Cibicidoides wuellerstorfi & 3.44 & 0.78 \\
\hline 38.43 & Cibicidoides wuellerstorfi & 2.93 & 1.17 \\
\hline
\end{tabular}

Table 1. (Continued).

\begin{tabular}{|c|c|c|c|}
\hline $\begin{array}{l}\text { Depth } \\
\text { (m) }\end{array}$ & Species & $\delta^{18} \mathrm{O}$ & $\delta^{13} \mathrm{C}$ \\
\hline 38.55 & Uvigerina sp. & 3.56 & 0.20 \\
\hline 38.64 & Uvigerina sp. & 3.83 & -0.10 \\
\hline 38.64 & Cibicidoides wuellerstorfi & 3.19 & 0.98 \\
\hline 38.75 & Uvigerina sp. & 3.80 & 0.14 \\
\hline 38.84 & Cibicidoides spp. & 2.63 & 0.72 \\
\hline 39.00 & Uvigerina sp. & 3.81 & -0.22 \\
\hline 39.10 & Uvigerina sp. & 3.76 & -0.12 \\
\hline 39.20 & Uvigerina sp. & 3.79 & -0.04 \\
\hline 39.30 & Cibicidoides wuellerstorfi & 3.21 & 1.22 \\
\hline 39.40 & Cib. and Melonis spp. & 3.18 & 0.51 \\
\hline 39.50 & Cib. and Melonis spp. & 3.31 & 0.28 \\
\hline 39.50 & Uvigerina and Gyroidina spp. & 3.83 & -0.43 \\
\hline 39.60 & Cibicidoides wuellerstorfi & 3.31 & 0.84 \\
\hline 39.70 & Cibicidoides wuellerstorfi & 3.42 & 0.77 \\
\hline 39.80 & Cibicidoides wuellerstorfi & 2.98 & 0.90 \\
\hline 39.90 & Cibicidoides wuellerstorfi & 2.86 & 0.60 \\
\hline 40.00 & Stilostomella spp. & 3.90 & -0.11 \\
\hline 40.10 & Uvigerina and mixed spp. & 3.08 & 0.48 \\
\hline 40.20 & Cibicidoides spp. & 2.57 & 0.67 \\
\hline 40.30 & Uvigerina and mixed spp. & 3.57 & -0.30 \\
\hline 40.30 & Cib. and Melonis spp. & 3.26 & 0.05 \\
\hline 40.40 & Cibicidoides wuellerstorfi & 2.96 & 1.13 \\
\hline 40.50 & mixed spp. & 3.24 & 0.21 \\
\hline 40.60 & Cibicidoides wuellerstorfi & 2.89 & 1.07 \\
\hline 40.70 & Uvigerina sp. & 4.02 & -0.19 \\
\hline 40.80 & Oridorsalis umbonifera & 4.56 & -0.50 \\
\hline 40.90 & Oridorsalis umbonifera & 4.50 & -0.43 \\
\hline 41.00 & Melonis spp. & 3.71 & -0.35 \\
\hline 41.10 & Cibicidoides spp. & 2.69 & 0.95 \\
\hline 41.20 & Cibicidoides wuellerstorfi & 2.85 & 0.68 \\
\hline 41.30 & Globocassidulina subglobosa & 4.66 & 0.07 \\
\hline 41.40 & Globocassidulina subglobosa & 4.95 & 0.15 \\
\hline 41.50 & Globocassidulina subglobosa & 4.08 & 0.16 \\
\hline 41.60 & Globocassidulina subglobosa & 4.31 & -0.01 \\
\hline 41.70 & Globocassidulina subglobosa & 4.54 & 0.29 \\
\hline 41.80 & Globocassidulina subglobosa & 4.90 & 0.24 \\
\hline 41.90 & Globocassidulina subglobosa & 4.72 & 0.14 \\
\hline 42.00 & Globocassidulina subglobosa & 4.85 & 0.16 \\
\hline 42.10 & Globocassidulina subglobosa & 4.67 & -0.23 \\
\hline 42.20 & Globocassidulina subglobosa & 4.00 & -0.10 \\
\hline 42.20 & Uvigerina sp. & 3.65 & -0.23 \\
\hline 42.30 & Uvigerina sp. & 3.48 & 0.15 \\
\hline 42.40 & Globocassidulina subglobosa & 3.19 & 0.48 \\
\hline 42.50 & Uvigerina $\mathrm{sp}$ & 3.43 & 0.11 \\
\hline 42.60 & Uvigerina $\mathrm{sp}$. & 3.56 & 0.07 \\
\hline 42.70 & Uvigerina sp. & 3.71 & 0.31 \\
\hline 42.80 & Globocassidulina subglobosa & 3.64 & 0.63 \\
\hline 42.90 & Globocassidulina subglobosa & 3.58 & 0.65 \\
\hline 43.00 & Globocassidulina subglobosa & 3.79 & 0.72 \\
\hline 43.10 & Globocassidulina subglobosa & 3.77 & 0.79 \\
\hline 43.20 & Globocassidulina subglobosa & 3.42 & 0.77 \\
\hline 43.30 & Globocassidulina subglobosa & 3.44 & 0.60 \\
\hline 43.40 & Globocassidulina subglobosa & 3.89 & 0.55 \\
\hline 43.50 & Globocassidulina subglobosa & 4.27 & 0.23 \\
\hline 43.60 & Uvigerina sp. & 3.21 & 0.33 \\
\hline 43.70 & Uvigerina sp. & 3.28 & 0.25 \\
\hline 43.80 & Uvigerina sp. & 3.54 & -0.10 \\
\hline 43.80 & Globocassidulina subglobosa & 3.78 & 0.55 \\
\hline 43.80 & Globocassidulina subglobosa & 3.95 & 0.43 \\
\hline 43.90 & Uvigerina sp. & 3.79 & 0.18 \\
\hline 44.00 & Globocassidulina subglobosa & 3.58 & 0.56 \\
\hline 44.10 & Uvigerina sp. & 3.48 & 0.13 \\
\hline 44.20 & Globocassidulina subglobosa & 3.58 & 0.54 \\
\hline 44.30 & Globocassidulina subglobosa & 3.89 & 0.40 \\
\hline 44.40 & Cibicidoides wuellerstorfi & 2.77 & 1.23 \\
\hline 44.50 & Cibicidoides wuellerstorfi & 2.90 & 1.17 \\
\hline 44.60 & Cibicidoides wuellerstorfi & 2.86 & 1.14 \\
\hline 44.70 & Uvigerina sp. & 3.34 & 0.31 \\
\hline 44.80 & Uvigerina sp. & 3.20 & 0.24 \\
\hline
\end{tabular}


Table 1. (Continued).

\begin{tabular}{|c|c|c|c|}
\hline $\begin{array}{l}\text { Depth } \\
\text { (m) }\end{array}$ & Species & $\delta^{18} \mathrm{O}$ & $\delta^{13} \mathrm{C}$ \\
\hline 44.90 & Uvigerina sp. & 3.26 & 0.34 \\
\hline 45.00 & Uvigerina sp. & 3.34 & 0.27 \\
\hline 45.10 & Uvigerina sp. & 3.57 & 0.36 \\
\hline 45.20 & Uvigerina sp. & 3.33 & 0.45 \\
\hline 45.30 & Globocassidulina subglobosa & 3.52 & 0.62 \\
\hline 45.40 & Uvigerina sp. & 3.64 & 0.25 \\
\hline 45.50 & Uvigerina sp. & 3.45 & 0.22 \\
\hline 45.53 & Globocassidulina subglobosa & 3.69 & 0.54 \\
\hline 45.70 & Uvigerina sp. & 3.28 & 0.22 \\
\hline 45.80 & Globocassidulina subglobosa & 3.49 & 0.51 \\
\hline 45.90 & Uvigerina sp. & 3.61 & -0.39 \\
\hline 45.90 & Globocassidulina subglobosa & 3.69 & 0.49 \\
\hline 45.90 & Globocassidulina subglobosa & 3.72 & 0.36 \\
\hline 45.90 & Uvigerina sp. & 3.83 & -0.07 \\
\hline 45.90 & Uvigerina sp. & 3.66 & -0.34 \\
\hline 46.00 & Globocassidulina subglobosa & 3.75 & 0.20 \\
\hline 46.10 & Cibicidoides wuellerstorfi & 2.62 & 1.10 \\
\hline 46.20 & Cibicidoides wuellerstorf $i$ & 2.69 & 0.87 \\
\hline 46.30 & Cibicidoides wuellerstorfi & 2.85 & 1.17 \\
\hline 46.40 & Globocassidulina subglobosa & 3.60 & 0.62 \\
\hline 46.50 & Cibicidoides wuellerstorfi & 2.67 & 0.97 \\
\hline 46.60 & Globocassidulina subglobosa & 3.61 & 0.58 \\
\hline 46.70 & Globocassidulina subglobosa & 3.60 & 0.54 \\
\hline 46.80 & Uvigerina sp. & 3.54 & -0.08 \\
\hline 46.80 & Globocassidulina subglobosa & 3.84 & 0.46 \\
\hline 46.90 & Globocassidulina subglobosa & 3.79 & 0.38 \\
\hline 47.00 & Globocassidulina subglobosa & 3.65 & 0.19 \\
\hline 47.10 & Cibicidoides wuellerstorfi & 2.91 & 1.04 \\
\hline 47.20 & Globocassidulina subglobosa & 3.39 & 0.34 \\
\hline 47.30 & Globocassidulina subglobosa & 3.50 & 0.21 \\
\hline 47.40 & Globocassidulina subglobosa & 3.52 & 0.42 \\
\hline 47.50 & Globocassidulina subglobosa & 3.31 & 0.57 \\
\hline 47.60 & Cibicidoides wuellerstorfi & 2.58 & 0.97 \\
\hline 47.70 & Cibicidoides wuellerstorfi & 2.64 & 1.15 \\
\hline 47.80 & Globocassidulina subglobosa & 3.32 & 0.52 \\
\hline 47.90 & Globocassidulina subglobosa & 2.89 & 0.33 \\
\hline 48.00 & Globocassidulina subglobosa & 3.33 & 0.55 \\
\hline 48.10 & Globocassidulina subglobosa & 3.47 & 0.43 \\
\hline 48.20 & Cibicidoides wuellerstorfi & 2.63 & 0.97 \\
\hline 48.30 & Uvigerina sp. & 3.10 & 0.07 \\
\hline 48.40 & Uvigerina sp. & 3.31 & 0.23 \\
\hline 48.50 & Cibicidoides wuellerstorf $i$ & 2.76 & 0.88 \\
\hline 48.60 & Uvigerina sp. & 3.45 & 0.04 \\
\hline 48.70 & Uvigerina $\mathrm{sp}$. & 3.16 & -0.07 \\
\hline 48.80 & Cibicidoides wuellerstorfi & 2.56 & 1.02 \\
\hline 48.80 & Uvigerina $\mathrm{sp}$. & 3.31 & 0.47 \\
\hline 48.90 & Uvigerina sp. & 2.95 & 0.23 \\
\hline 49.00 & Uvigerina sp. & 3.03 & 0.18 \\
\hline 49.03 & Uvigerina $\mathrm{sp}$ & 3.59 & 0.06 \\
\hline 49.10 & Cibicidoides wuellerstorfi & 2.60 & 0.84 \\
\hline 49.20 & Cibicidoides wuellerstorfi & 3.08 & 1.08 \\
\hline 49.30 & Uvigerina sp. & 3.47 & 0.32 \\
\hline 49.40 & Cibicidoides wuellerstorfi & 2.55 & 0.99 \\
\hline 49.50 & Cibicidoides wuellerstorfi & 2.49 & 0.89 \\
\hline 49.70 & Uvigerina sp. & 3.41 & 0.15 \\
\hline 49.80 & Uvigerina sp. & 2.87 & 0.24 \\
\hline 49.90 & Uvigerina sp. & 2.98 & 0.39 \\
\hline 50.00 & Uvigerina $\mathrm{sp}$. & 2.77 & 0.17 \\
\hline 50.10 & Uvigerina $\mathrm{sp}$. & 3.02 & 0.12 \\
\hline 50.20 & Uvigerina $\mathrm{sp}$. & 3.39 & 0.40 \\
\hline 50.30 & Uvigerina sp. & 2.76 & 0.04 \\
\hline 50.40 & Uvigerina $\mathrm{sp}$. & 2.67 & 0.02 \\
\hline 50.50 & Uvigerina sp. & 2.63 & 0.79 \\
\hline 50.60 & Cibicidoides wuellerstorfi & 2.59 & 1.02 \\
\hline 50.70 & Cibicidoides wuellerstor $f i$ & 2.42 & 0.63 \\
\hline 50.80 & Oridorsalis umbonifera & 2.67 & 0.85 \\
\hline 50.80 & Sphaeroidina bulloides & 3.18 & 0.24 \\
\hline 50.90 & Globocassidulina subglobosa & 3.29 & -0.49 \\
\hline 51.00 & Cibicidoides spp. & 2.54 & 0.67 \\
\hline
\end{tabular}

Table 1. (Continued).

\begin{tabular}{|c|c|c|c|}
\hline $\begin{array}{c}\text { Depth } \\
\text { (m) }\end{array}$ & Species & $\delta^{18} \mathrm{O}$ & $\delta^{13} \mathrm{C}$ \\
\hline 51.10 & Globocassidulina subglobosa & 3.45 & 0.20 \\
\hline 51.10 & Globocassidulina subglobosa & 3.88 & -0.00 \\
\hline 51.20 & Globocassidulina subglobosa & 3.44 & 0.02 \\
\hline 51.30 & Globocassidulina subglobosa & 3.65 & 0.09 \\
\hline 51.40 & Uvigerina sp. & 3.50 & 0.17 \\
\hline 51.50 & Globocassidulina subglobosa & 3.22 & 0.10 \\
\hline 51.60 & Cibicidoides spp. & 3.00 & 0.78 \\
\hline 51.60 & Globocassidulina subglobosa & 3.50 & 0.44 \\
\hline 51.70 & Globocassidulina subglobosa & 3.22 & 0.46 \\
\hline 51.80 & Globocassidulina subglobosa & 3.12 & 0.28 \\
\hline 51.90 & Globocassidulina subglobosa & 3.20 & 0.17 \\
\hline 52.00 & Uvigerina sp. & 3.29 & 0.25 \\
\hline 52.10 & Cibicidoides wuellerstorfi & 2.25 & -0.30 \\
\hline 52.20 & Cibicidoides wuellerstorf $i$ & 2.38 & 0.76 \\
\hline 52.30 & Globocassidulina subglobosa & 3.36 & 0.14 \\
\hline 52.30 & Cibicidoides wuellerstorfi & 2.85 & 0.87 \\
\hline 52.40 & Cibicidoides wuellerstorfi & 2.70 & 0.74 \\
\hline 52.50 & Globocassidulina subglobosa & 3.53 & 0.30 \\
\hline 52.60 & Cibicidoides wuellerstorfi & 2.18 & 0.70 \\
\hline 52.70 & Cibicidoides wuellerstorfi & 2.38 & 0.90 \\
\hline 52.80 & Cibicidoides wuellerstorf $i$ & 2.62 & 0.86 \\
\hline 52.90 & Globocassidulina subglobosa & 3.32 & 0.27 \\
\hline 53.00 & Cibicidoides wuellerstorf $i$ & 2.44 & 0.62 \\
\hline 53.10 & Cibicidoides wuellerstorfi & 2.32 & 0.45 \\
\hline 53.20 & Globocassidulina subglobosa & 3.02 & 0.28 \\
\hline 53.30 & Cibicidoides kullenbergi & 2.70 & 0.35 \\
\hline 53.30 & Globocassidulina subglobosa & 3.46 & 0.23 \\
\hline 53.40 & Globocassidulina subglobosa & 3.19 & 0.42 \\
\hline 53.50 & Globocassidulina subglobosa & 3.29 & 0.16 \\
\hline 53.60 & Cibicidoides wuellerstorfi & 2.49 & 0.61 \\
\hline 53.70 & Cibicidoides wuellerstorfi & 2.41 & 0.62 \\
\hline 53.80 & Cibicidoides wuellerstorfi & 2.35 & 0.73 \\
\hline 53.90 & Cibicidoides wuellerstorfi & 2.65 & 0.95 \\
\hline 54.00 & Cibicidoides wuellerstorfi & 2.33 & 0.70 \\
\hline 54.00 & Globocassidulina subglobosa & 3.04 & 0.50 \\
\hline 54.00 & Cibicidoides wuellerstorf $i$ & 2.66 & 0.89 \\
\hline 54.10 & Cibicidoides wuellerstorfi & 2.57 & 1.13 \\
\hline 54.20 & Uvigerina sp. & 2.92 & 0.65 \\
\hline 54.30 & Cibicidoides wuellerstorfi & 2.56 & 1.19 \\
\hline 54.40 & Globocassidulina subglobosa & 3.07 & 0.68 \\
\hline 54.50 & Cibicidoides wuellerstorfi & 2.62 & 1.08 \\
\hline 54.60 & Globocassidulina subglobosa & 3.22 & 0.59 \\
\hline 54.70 & Globocassidulina subglobosa & 3.20 & 0.70 \\
\hline 54.80 & Globocassidulina subglobosa & 3.13 & 0.63 \\
\hline 54.90 & Globocassidulina subglobosa & 3.14 & 0.54 \\
\hline 55.00 & Uvigerina sp. & 2.91 & 0.31 \\
\hline 55.10 & Cibicidoides wuellerstorfi & 2.56 & 0.79 \\
\hline 55.20 & Uvigerina sp. & 3.03 & 0.20 \\
\hline 55.30 & Globocassidulina subglobosa & 3.45 & 0.38 \\
\hline 55.30 & Globocassidulina subglobosa & 3.33 & 0.35 \\
\hline 55.30 & Cibicidoides wuellerstorf $t$ & 2.58 & 0.96 \\
\hline 55.40 & Cibicidoides wuellerstorfi & 2.47 & 0.98 \\
\hline 55.50 & Cibicidoides wuellerstorfi & 2.63 & 1.10 \\
\hline 55.60 & Globocassidulina subglobosa & 3.27 & 0.60 \\
\hline 55.70 & Cibicidoides wuellerstorfi & 2.48 & 1.03 \\
\hline 55.80 & Cibicidoides wuellerstorf $i$ & 2.55 & 1.11 \\
\hline 55.90 & Uvigerina and $G$. subglobosa & 3.10 & 0.54 \\
\hline 55.90 & Cibicidoides spp. & 2.32 & 1.02 \\
\hline 56.00 & Cibicidoides kullenbergi & 2.42 & 0.99 \\
\hline 56.10 & Cibicidoides wuellerstorfi & 2.52 & 1.20 \\
\hline 56.20 & Globocassidulina subglobosa & 3.20 & 0.61 \\
\hline 56.30 & Globocassidulina subglobosa & 3.21 & 0.61 \\
\hline 56.40 & Cibicidoides wuellerstorfi & 2.54 & 1.18 \\
\hline 56.50 & Cibicidoides wuellerstorfi & 2.29 & 1.05 \\
\hline 56.60 & Cibicidoides wuellerstorfi & 2.53 & 0.90 \\
\hline 56.70 & Uvigerina sp. & 3.07 & 0.22 \\
\hline 56.80 & Uvigerina sp. & 3.27 & 0.29 \\
\hline 56.90 & Uvigerina sp. & 3.37 & 0.43 \\
\hline 57.00 & Globocassidulina subglobosa & 3.13 & 0.35 \\
\hline
\end{tabular}


Table 1. (Continued).

\begin{tabular}{clrr}
\hline $\begin{array}{c}\text { Depth } \\
(\mathrm{m})\end{array}$ & \multicolumn{1}{c}{ Species } & $\delta^{18} \mathrm{O}$ & $\delta^{13} \mathrm{C}$ \\
\hline 57.10 & Globocassidulina subglobosa & 3.39 & 0.51 \\
57.20 & Uvigerina sp. & 3.17 & 0.37 \\
57.20 & Globocassidulina subglobosa & 3.34 & 0.63 \\
57.30 & Cibicidoides wuellerstorfi & 2.44 & 1.16 \\
57.40 & Cibicidoides wuellerstor $f i$ & 2.44 & 1.19 \\
57.50 & Uvigerina sp. & 2.95 & 0.12 \\
57.60 & Uvigerina sp. & 3.13 & 0.26 \\
57.70 & Uvigerina sp. & 3.38 & 0.29 \\
57.80 & Uvigerina sp. & 3.42 & 0.20 \\
57.90 & Uvigerina sp. & 3.29 & 0.08 \\
57.90 & Globocassidulina subglobosa & 3.64 & 0.56 \\
58.00 & Uvigerina sp. & 3.58 & 0.08 \\
58.10 & Uvigerina sp. & 3.25 & -0.03 \\
58.20 & Uvigerina sp. & 3.11 & 0.15 \\
58.30 & Uvigerina sp. & 2.98 & 0.33 \\
58.40 & Uvigerina sp. & 2.81 & 0.56 \\
58.50 & Cibicidoides wuellerstor $f i$ & 2.37 & 1.16 \\
58.60 & Uvigerina sp. & 2.76 & 0.83 \\
58.70 & Cibicidoides wuellerstor $f i$ & 2.53 & 1.19 \\
58.80 & Cibicidoides wuellerstor $f i$ & 2.43 & 1.14 \\
58.90 & Cibicidoides wuellerstor $f i$ & 2.52 & 0.94 \\
58.97 & Sphaeroidina bulloides & 3.13 & 0.21 \\
58.97 & Cibicidoides wuellerstorf $i$ & 2.47 & 1.09 \\
\hline & & & \\
\hline
\end{tabular}

analysis of benthic species. One feature of the record that certainly requires further study is that there are many interglacial peaks that do not attain the expected value of about $+3.2 \%$, although the accumulation rate appears high enough that the extremes should be well represented.

Below this point, the record is less well known. In Figure 3 the record for Cores 7 to 12 (below disturbed Core 6) is compared with that of core V28-179 (Shackleton and Opdyke, 1977); this is the only published ben-
Table 2. Oxygen and carbon isotope adjustment factors used.

\begin{tabular}{lrr}
\hline \multicolumn{1}{c}{ Species } & \multicolumn{1}{c}{${ }^{18} \mathrm{O}$} & \multicolumn{1}{c}{${ }^{13} \mathrm{C}$} \\
\hline Uvigerina peregrina & \multicolumn{1}{c}{0.0} & 0.9 \\
Cibicidoides wuellerstorfi & 0.64 & 0.0 \\
Globocassidulina subglobosa & -0.1 & 0.5 \\
Hoeglundina elegans & -0.4 & -1.3 \\
Melonis pompiliodes & 0.3 & 0.6 \\
Melonis barleeanum & 0.3 & 1.0 \\
Oridorsalis umbonifera & 0.0 & 1.0 \\
Sphaeroidina bulloides & -0.1 & -0.1 \\
Stilostomella sp. & -0.15 & 1.0 \\
\hline
\end{tabular}

thic ${ }^{18} \mathrm{O}$ record covering the time interval back to 3.5 m.y. ago (the same species adjustment has been made for these analyses as for DSDP Hole 552A). It is very clear that the isotopic range is greater for Hole 552A than for core V28-179. The chief reason for this must be the very low accumulation rate of only about $0.55 \mathrm{~cm} / \mathrm{k} . \mathrm{y}$. in V28-179; abundant experience with Pleistocene cores shows that it is very difficult to obtain a correlatable ${ }^{18} \mathrm{O}$ record in regions with an accumulation rate significantly less than $1 \mathrm{~cm} / \mathrm{k} . \mathrm{y}$.

Although no high-resolution records of deep-water ${ }^{18} \mathrm{O}$ changes in the Pacific are yet available for the early Pleistocene, it seems rather likely that Hole 552A will prove to preserve a significant record of deep-water temperature variability in addition to an ice volume record, just as is now known to be the case for late Pleistocene records from the North Atlantic (Shackleton, Imbrie, and Hall, 1983). However, even if this proves to be the case, the glacial event at about 2.4 m.y. ago must certainly have been more severe than was recognized by Shackleton and Opdyke (1977), with ${ }^{18} \mathrm{O}$ values almost

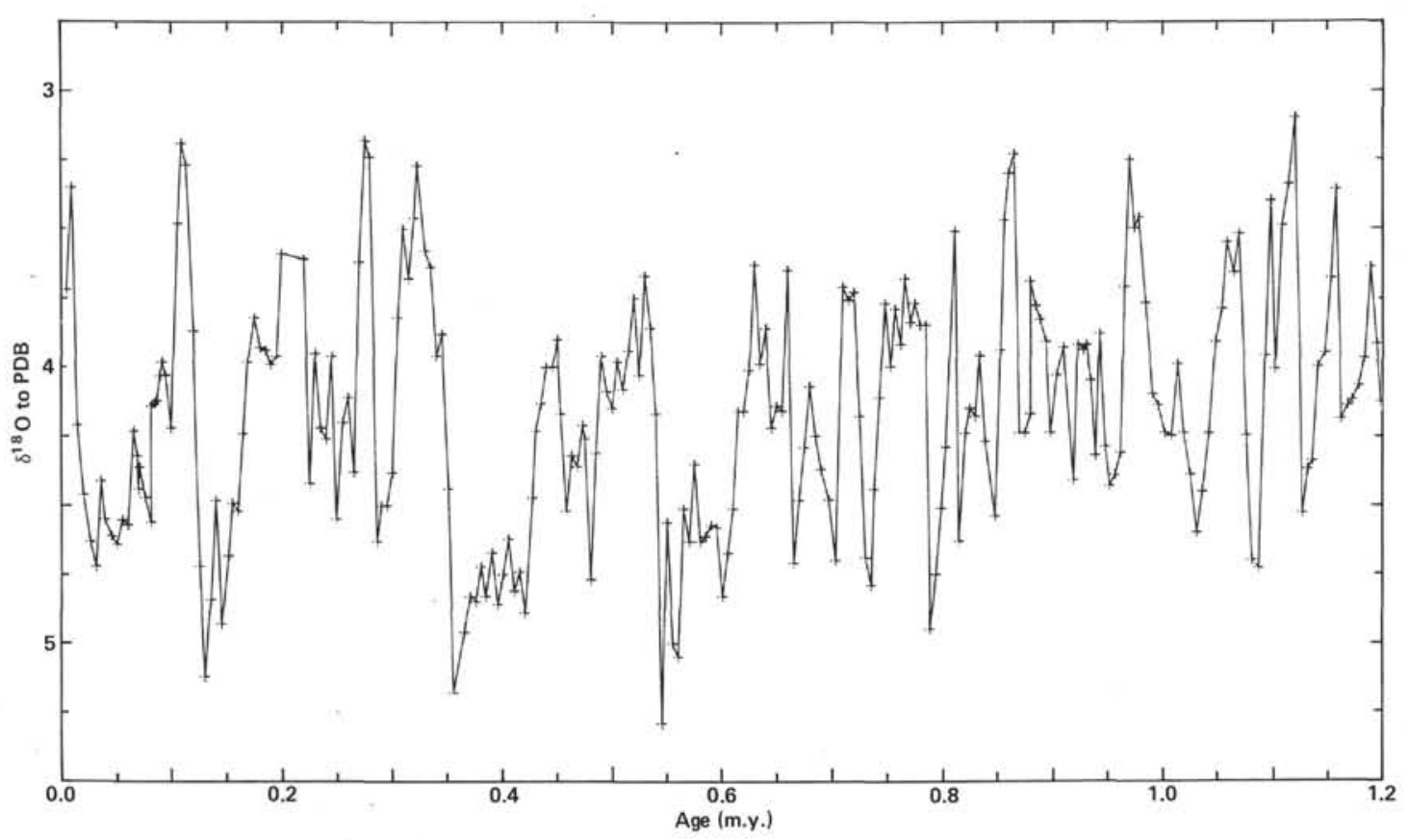

Figure 2. Oxygen isotope data for Hole 552A (Cores 1 to 5) plotted on an age scale (from Table 3). 


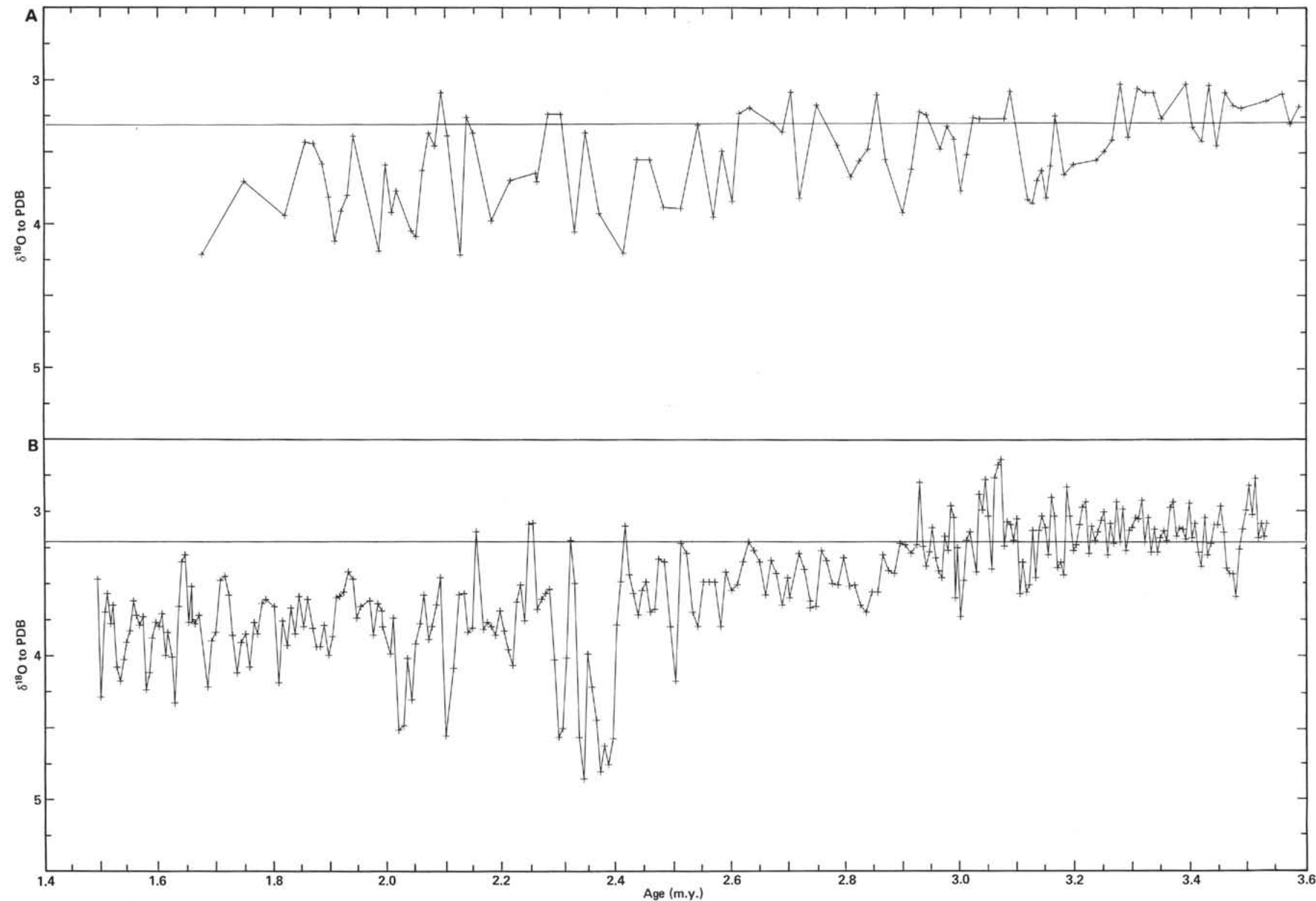

Figure 3. A. Oxygen isotope data for piston core V28-179 (Shackleton and Opdyke, 1977). B. Oxygen isotope data for Hole 552A plotted on an age scale (from Table 3); lower part, Cores 7 to 12. Horizontal lines are drawn at about the position of equilibrium ${ }^{18} \mathrm{O}$ in today's conditions. 


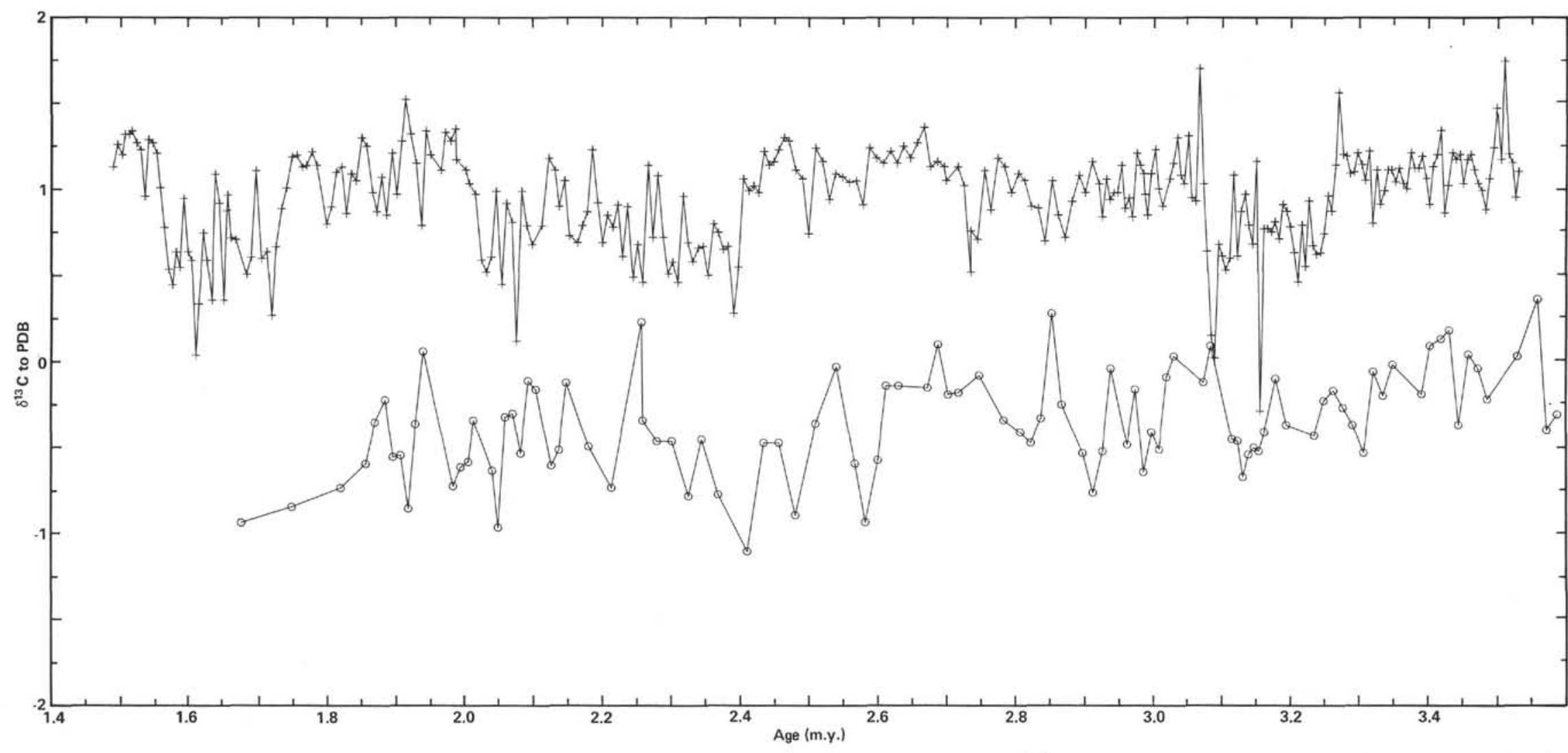

Figure 4. Carbon isotope data for Hole 552A (Atlantic, crosses) and for core V28-179 (Pacific, circles) plotted on the same ${ }^{13} \mathrm{C}$ and age scales. The persistent of fset between North Atlantic and equatorial Pacific values shows that a water mass similar to NADW was present on Rockall throughout the interval represented. 
Table 3. Age control points used for time scale construction.

\begin{tabular}{ccl}
\hline $\begin{array}{c}\text { Depth } \\
(\mathrm{m})\end{array}$ & $\begin{array}{c}\text { Age } \\
(\mathrm{m} . \mathrm{y})\end{array}$ & \multicolumn{1}{c}{ Identification } \\
\hline 0.0 & 0.0 & Core top \\
14.60 & 0.73 & Base Brunhes chron \\
20.10 & 0.98 & Base Jaramillo subchron \\
32.10 & 1.66 & Top Olduvai subchron \\
43.20 & 2.47 & Top Gauss chron \\
47.80 & 2.99 & Base Kaena subchron \\
56.60 & 3.40 & Base Gauss chron \\
\hline
\end{tabular}

as positive as those in the upper part of the record (Fig. 2). This glacial event must represent the major environmental event in the late Neogene of northwest Europe, although it was almost a million years before the Pliocene/Pleistocene boundary (Backman et al., 1983).

The record from below the glacial event at 2.37 m.y. ago shows frequent ${ }^{18} \mathrm{O}$ values that deviate in the isotopically light direction with reference to the present equilibrium values of about $3.2 \%$ (whereas middle Pleistocene interglacial peaks generally only just attain this level). This might be interpreted in terms of deepwater temperature variations in the water covering Rockall between present-day values and up to 2 degrees warmer than this. However, in Figure 3 lines are drawn to indicate the present equilibrium value for both the Hole 552A record and that from the deep Pacific; examination of the deviations from these lines reveals that the excursions in the isotopically light direction are common to both records, so that they could equally be interpreted in terms of partial deglaciation of Antarctica. Further work is needed to distinguish between these alternatives, and in particular a high-resolution record from the deep Pacific is needed.

\section{CARBON ISOTOPE RECORD}

The carbon isotope gradients that exist within the ocean deep waters are an important guide to ocean circulation patterns (Kroopnick, 1980). A water mass forming at the surface has the ${ }^{13} \mathrm{C}$ content of surface water and a high dissolved oxygen content. During the deep water ageing of this water mass, dissolved oxygen is utilized by the oxidation of organic matter that sinks from the sea surface, and the dissolved $\mathrm{CO}_{2}$ becomes isotopically lighter as a consequence of the addition of this isotopically light carbon.

Figure 4 compares the ${ }^{13} \mathrm{C}$ records of Hole $552 \mathrm{~A}$ and piston core V28-179. Note that the published data for core V28-179 (Shackleton and Opdyke, 1977), and some additional measurements, have been adjusted for species-effects on the basis of Table 2, so that the difference between the two data sets in Figure 4 should be a measure of the ${ }^{13} \mathrm{C}$ difference between the two water masses. It is clear from this comparison that the water mass bathing Rockall was younger than that of the deep Pacific throughout the interval studied. Moreover, the high-frequency variability present was approximately the same prior to the major climatic event at $2.4 \mathrm{~m}$.y. ago as after; that is to say, the ${ }^{13} \mathrm{C}$ variability was not forced by glacial-interglacial change. High-resolution stratigraphic correlation through the use of ${ }^{13} \mathrm{C}$ variability may ultimately be possible in those parts of the record lacking sufficient ${ }^{18} \mathrm{O}$ variability for this purpose.

\section{PLANKTONIC DATA}

Table 4 lists data for Globigerina atlantica in the section below the lowest ice-rafting horizon. Some difficulty was experienced in picking an identical morphology through the interval. However, the data seem not to give any indication of significant surface temperature changes over this time interval; the range of variation is about the same as in benthic species. Since nothing is known of the depth habitat of $G$. atlantica, this observation may prove to have no bearing on true surface temperature variations. Recently Fairbanks et al. (1982) showed that in the Pacific, $N$. dutertrei calcifies at more or less the same temperature throughout the year. It is possible that in an analogous manner $G$. atlantica adjusted its preferred depths habitat over the interval studied so as to maintain a constant temperature. We are at present unable to resolve this question.

\section{CONCLUSIONS}

Oxygen isotope analysis in Hole 552A demonstrates that a major glacial event occurred about $2.37 \mathrm{~m} . \mathrm{y}$ ago, almost a million years before the Pliocene/Pleistocene boundary. The oxygen isotope record above this event represents an exceptionally detailed ice volume record marred only by one disturbed core. Had the site been double-cored it would have been essentially an ideal record. A detailed record of temperature variations below this event will be better understood when records of comparable temporal resolution have been obtained from the deep Pacific. Carbon isotope analysis shows that North Atlantic Deep Water (NADW) existed at least back to 3.5 m.y. ago, but that the quasi-cyclic alternations in the intensity of its production were occurring even during the Pliocene before the onset of glaciation.

\section{ACKNOWLEDGMENTS}

We are very grateful to the scientific crew of Leg 81 for making these samples available to us, and especially to Jan Backman and Herman Zimmerman for the time they devoted to taking and preparing samples as well as to reviewing the manuscript. Dennis Kent generously shared his magnetic data with us; Jan Backman was equally generous with his biostratigraphic data. Nick Pisias, John Imbrie, and Pierre Pestiaux kept up our production by showing their enthusiasm for the data. In Cambridge, C. Solanki and M. Tabecki contributed a great deal to the sample processing. We are grateful to NERC for support of the laboratory in Cambridge through grant GR3/3162, as well as for their support of DSDP.

\section{REFERENCES}

Backman, J., Tauxe, L., and Shackleton, N. J., 1983. Quantitative nannofossil correlation to open ocean deep sea sections from the Plio-Pleistocene boundary at Vrica, Italy. Nature, 304:156-158.

Fairbanks, R. G., Sverdlove, M., Free, R., Wiebe, P. H., and Bé, A. W. H., 1982. Vertical distribution and isotopic fractionation of living planktonic Foraminifera from the Panama Basin. Nature, 289: 841-844.

Kroopnick, P., 1980. The distribution of ${ }^{13} \mathrm{C}$ in the Atlantic Ocean. Earth Planet. Sci. Lett., 49:469-484.

Ruddiman, W. F., and McIntyre, A., 1976. Northeast Atlantic paleoclimatic changes over the past 600,000 years. Mem. Geol. Soc. Am., 145:111-146. 
Shackleton, N. J., Backman, J., Zimmerman, H., Kent, D. V., Hall, M. A., Roberts, D. G., Schnitker, D., Baldauf, J. G., Desprairies, A., Homrighausen, R., Huddlestun, P., Keene, J. B., Kaltenbach, A. J., Krumsiek, K. A. O., Morton, A. C., Murray, J. W., and Westberg-Smith, J., 1984. Oxygen isotope calibration of the onset of ice-rafting and history of glaciation in the North Atlantic region. Nature, 307:620-623.

Shackleton, N. J., and Hall, M. A., 1983. Stable isotope record of Hole 504 sediments: High resolution record of the Pleistocene. In Cann, J. R., Langseth, M. G., et al., Init. Repts. DSDP, 69: Washington (U.S. Govt. Printing Office), 431-441.

Shackleton, N. J., Imbrie, J., and Hall, M. A., 1983. Oxygen and carbon isotope record of East Pacific core V19-30: Implications for the formation of deep water in the late Pleistocene North Atlantic. Earth Planet. Sci. Lett., 65:233-244.

Shackleton, N. J., and Opdyke, N. D., 1973. Oxygen isotope and palaeomagnetic stratigraphy of equatorial Pacific core V28-238: Oxygen isotope temperatures and ice volumes on a $10^{5}$ year and $10^{6}$ year scale. Quat. Res., 3:39-55. 1977. Oxygen isotope and palaeomagnetic evidence for early Northern Hemisphere glaciation. Nature, 270:216-219.

Date of Acceptance: August 22, 1983

Table 4. Oxygen and carbon isotope data for Globigerina atlantica.

\begin{tabular}{ccrrrr}
\hline $\begin{array}{c}\text { Depth } \\
(\mathrm{m})\end{array}$ & $\delta^{18} \mathrm{O}$ & $\delta^{13} \mathrm{C}$ & $\begin{array}{c}\text { Depth } \\
(\mathrm{m})\end{array}$ & $\delta^{18} \mathrm{O}$ & $\delta^{13} \mathrm{C}$ \\
\hline 42.70 & 2.01 & 0.70 & 50.50 & 1.86 & 0.68 \\
42.80 & 2.24 & 0.74 & 50.60 & 1.82 & 0.43 \\
42.90 & 1.92 & 0.81 & 50.70 & 2.00 & 0.37 \\
43.0 & 2.18 & 0.84 & 50.80 & 2.05 & 0.23 \\
43.10 & 1.94 & 0.86 & 50.90 & 2.21 & 0.03 \\
43.20 & 1.93 & 0.92 & 51.00 & 1.75 & 0.13 \\
43.30 & 1.92 & 0.80 & 51.10 & 2.20 & -0.27 \\
43.40 & 2.80 & 0.99 & 51.20 & 2.18 & -0.18 \\
43.50 & 2.57 & 0.94 & 51.30 & 1.91 & -0.19 \\
43.60 & 2.29 & 0.75 & 51.40 & 1.96 & -0.18 \\
43.70 & 2.07 & 0.97 & 51.50 & 1.98 & 0.02 \\
43.70 & 1.67 & -0.04 & 51.60 & 2.31 & -0.19 \\
43.80 & 2.19 & 0.83 & 51.70 & 1.70 & 0.04 \\
43.90 & 1.85 & 0.69 & 51.80 & 1.94 & 0.24 \\
44.00 & 2.20 & 0.74 & 51.90 & 1.88 & 0.15 \\
44.10 & 1.83 & 1.07 & 52.00 & 1.77 & -0.00 \\
44.20 & 1.94 & 1.01 & 52.10 & 1.92 & 0.32 \\
44.30 & 2.11 & 0.48 & 52.20 & 1.74 & 0.10 \\
44.40 & 2.14 & 1.08 & 52.30 & 1.61 & 0.11 \\
44.50 & 2.33 & 0.97 & 52.40 & 2.11 & 0.23 \\
44.60 & 2.32 & 0.86 & 52.50 & 1.99 & 0.24 \\
44.70 & 2.13 & 1.15 & 52.60 & 1.71 & 0.24 \\
44.80 & 2.14 & 0.93 & 52.70 & 1.75 & 0.04 \\
44.90 & 2.24 & 1.18 & 52.80 & 1.66 & 0.33 \\
45.00 & 2.07 & 1.03 & 52.90 & 1.69 & 0.17 \\
45.10 & 1.99 & 0.86 & 53.00 & 1.53 & 0.39 \\
\hline & & & & &
\end{tabular}

Table 4. (Continued).

\begin{tabular}{|c|c|c|c|c|c|}
\hline $\begin{array}{c}\text { Depth } \\
\text { (m) }\end{array}$ & $\delta^{18} \mathrm{O}$ & $\delta^{13} \mathrm{C}$ & $\begin{array}{l}\text { Depth } \\
\text { (m) }\end{array}$ & $\delta^{18} \mathrm{O}$ & $\delta^{13} \mathrm{C}$ \\
\hline 45.20 & 1.94 & 1.04 & 53.10 & 1.65 & 0.48 \\
\hline 45.30 & 2.00 & 0.93 & 53.20 & 1.93 & 0.43 \\
\hline 45.40 & 2.12 & 0.89 & 53.30 & 1.65 & 0.48 \\
\hline 45.50 & 1.95 & 0.85 & 53.40 & 2.00 & 0.53 \\
\hline 45.53 & 2.08 & 0.91 & 53.50 & 1.69 & 0.50 \\
\hline 45.70 & 1.94 & 0.97 & 53.60 & 1.77 & 0.47 \\
\hline 45.80 & 2.04 & 0.81 & 53.70 & 1.78 & 0.53 \\
\hline 45.90 & 2.33 & 0.83 & 53.80 & 1.57 & 0.60 \\
\hline 46.00 & 1.97 & 0.69 & 55.10 & 1.67 & 0.50 \\
\hline 46.10 & 1.96 & 1.04 & 55.20 & 1.83 & 0.29 \\
\hline 46.21 & 2.00 & 1.02 & 55.30 & 1.64 & 0.26 \\
\hline 46.30 & 2.10 & 0.95 & 55.40 & 1.93 & 0.17 \\
\hline 46.40 & 2.04 & 0.97 & 55.50 & 1.63 & 0.18 \\
\hline 46.50 & 2.17 & 0.93 & 55.60 & 1.60 & 0.21 \\
\hline 46.60 & 1.98 & 0.90 & 55.70 & 1.33 & 0.25 \\
\hline 46.70 & 2.25 & 0.91 & 55.80 & 1.32 & 0.23 \\
\hline 46.80 & 2.26 & 0.86 & 55.90 & 1.58 & 0.32 \\
\hline 46.90 & 2.20 & 1.09 & 56.00 & 1.46 & 0.33 \\
\hline 47.00 & 2.09 & 0.75 & 56.10 & 1.54 & 0.27 \\
\hline 47.10 & 1.86 & 0.80 & 56.20 & 1.51 & 0.58 \\
\hline 47.20 & 2.00 & 0.90 & 56.30 & 1.71 & 0.57 \\
\hline 47.30 & 1.96 & 0.71 & 56.30 & 1.41 & 0.36 \\
\hline 47.40 & 1.88 & 0.67 & 56.40 & 1.55 & 0.59 \\
\hline 47.50 & 2.01 & 0.79 & 56.40 & 1.44 & 0.37 \\
\hline 47.60 & 2.11 & 0.73 & 56.50 & 1.53 & 0.51 \\
\hline 47.70 & 1.80 & 0.88 & 56.50 & 1.50 & 0.35 \\
\hline 48.00 & 1.92 & 0.51 & 56.60 & 1.84 & 0.56 \\
\hline 48.10 & 1.89 & 0.71 & 56.60 & 1.46 & 0.06 \\
\hline 48.20 & 1.88 & 0.75 & 56.70 & 1.73 & 0.24 \\
\hline 48.30 & 2.08 & 0.85 & 56.80 & 1.67 & 0.16 \\
\hline 48.30 & 1.81 & 0.69 & 56.90 & 1.68 & 0.50 \\
\hline 48.40 & 2.19 & 0.65 & 57.00 & 1.78 & 0.68 \\
\hline 48.40 & 1.88 & 0.67 & 57.10 & 1.68 & 0.53 \\
\hline 48.50 & 2.22 & 0.90 & 57.20 & 1.78 & 0.38 \\
\hline 48.60 & 2.05 & 0.54 & 57.30 & 1.99 & 0.66 \\
\hline 48.70 & 2.24 & 0.67 & 57.40 & 1.84 & 0.66 \\
\hline 48.80 & 1.77 & 0.66 & 57.50 & 1.84 & 0.44 \\
\hline 48.81 & 1.95 & 0.90 & 57.60 & 1.85 & 0.26 \\
\hline 48.90 & 1.71 & 0.71 & 57.70 & 1.72 & 0.24 \\
\hline 49.00 & 2.07 & 0.81 & 57.80 & 1.84 & 0.16 \\
\hline 49.10 & 1.97 & 0.32 & 57.90 & 2.02 & 0.08 \\
\hline 49.20 & 2.20 & 0.85 & 58.00 & 1.92 & 0.13 \\
\hline 49.30 & 2.06 & 0.75 & 58.10 & 1.80 & 0.09 \\
\hline 49.40 & 1.92 & 0.86 & 58.20 & 1.75 & 0.35 \\
\hline 49.60 & 2.24 & 1.11 & 58.30 & 1.72 & 0.64 \\
\hline 49.70 & 2.31 & 1.06 & 58.40 & 1.65 & 0.74 \\
\hline 49.80 & 1.86 & 0.85 & 58.50 & 1.46 & 0.77 \\
\hline 49.90 & 1.78 & 0.86 & 58.60 & 1.63 & 1.14 \\
\hline 50.00 & 1.76 & 0.82 & 58.70 & 1.76 & 0.98 \\
\hline 50.10 & 1.70 & 0.26 & 58.80 & 1.72 & 0.79 \\
\hline 50.20 & 1.60 & 0.14 & 58.87 & 1.74 & 0.53 \\
\hline 50.30 & 1.48 & 0.13 & 58.97 & 1.82 & 0.70 \\
\hline
\end{tabular}

